\title{
OPTIMIZED CONVECTIVE VOLUME IN ONLINE
}

\section{HEMODIAFILTRATION}

\author{
František Lopot ${ }^{1,2}$, Vratislav Fabián ${ }^{3}$ \\ ${ }^{1}$ Department of Medicine, General University Hospital in Prague, Prague-Strahov, Czech Republic \\ ${ }^{2}$ Institute of Biophysics and Medical Informatics, First Faculty of Medicine, Charles University, \\ Prague, Czech Republic \\ ${ }^{3}$ Department of Physics, Faculty of Electrical Engineering, Czech Technical University in Prague, \\ Czech Republic
}

\begin{abstract}
Hemodiafiltration $(H D F)$ adds convective elimination of middle molecules $(M M)$, proportional to filtered volume ( $\left.V_{\text {conv }}\right)$ on the top of diffusion-based elimination of small molecules (SM) by conventional hemodialysis (HD). Studies, both observational and randomized controlled ones, performed so far generally indicated positive impact of higher $V_{\text {conv }}$ on all-cause mortality in HDF patients, although the magnitude of $V_{\text {conv }}$ at which HDF becomes apparently superior to HD differed widely among the studies. Also the issue of a suitable anthropometric parameter by which the $V_{\text {conv }}$ should be normalized has not yet been solved. Data from the ESHOL study seems to indicate that patient's body surface area (BSA) could well be used for this-mortality was decreasing with increasing $V_{\text {conv }} / B S A$ with a bottom plateau starting at about $15 \mathrm{~L} / \mathrm{m}^{2}$. We have therefore devised a formula and a graph for determination of $V_{\text {conv }}$ which fulfils the requirement $V_{\text {conv }} / B S A=15$. Assuming maximum feasible and safe filtration fraction $Q F / Q B=0.3$, the $V_{\text {conv }}$ actually defines the necessary blood flow $(Q B)$ to reach $V_{\text {conv }} / B S A=15$ as $Q B=15 \cdot B S A /(0.3 \cdot t)(t-$ session time). It is also possible to check what $Q B$ is needed in terms of sufficient diffusion-based transport (target Kt/V) and compare both $Q B$ values to see if adequate combined elimination of both SM and MM can be achieved at the same time and under what conditions, respectively.
\end{abstract}

\section{Keywords}

treatment adequacy, prescription, monitoring, hemodiafiltration, convective volume, body surface area, filtration fraction, blood flow, hemodialysis, combined approach

\section{Introduction}

Hemodiafiltration (HDF) adds convective elimination of middle molecules (MM) on the top of diffusionbased elimination of small molecules (SM) by conventional hemodialysis (HD). However, unlike the situation in SM range, where the "relative dialysis dose" $K t / V$ is generally accepted as its adequacy marker with its target value defined by randomized controlled studies $[1,2]$ and included in existing guidelines $[3,4]$ there is only a general consensus that the "absolute dialysis dose" in MM range may be represented by the convective volume $\left(V_{\text {conv }}\right)$. No consensus exists as to which anthropometric parameter should be used to convert that absolute dose into a relative one which would enable to compare HDF efficacy in different individuals. Consequently, also no target value for the absolute $V_{\text {conv }}$ and its relative value exists either. The result is widely varying prescription of HDF effectively hindering reliable comparison of its outcome reported by different groups. From among the plethora of studies on online HDF, only one study [5] provided some data for the search of a suitable anthropometric parameter applicable for $V_{c o n v}$ normalization needed to find its upper target value above which no further benefit for the patient could be expected. Indeed, $V_{\text {conv }}$ normalized by $B S A$ indicates that mortality of patients on HDF may be reaching its bottom plateau for values of $V_{\text {conv }} / B S A$ around $15 \mathrm{~L} / \mathrm{m}^{2}$.

Based on this estimation, this article devises computational and graphic approach for prescription of desirable $V_{\text {conv }}$ according to patient height and weight (parameters used to estimate his/her BSA). Assuming further reasonable and safe value of filtration fraction $F F=Q F / Q B=0.3(Q F-$ filtration rate, $Q B-$ blood 
flow during HDF), necessary $Q B$ is defined to reach the target value of "convective dose". Finally, the $Q B$ defined in this way is compared to the $Q B$ needed to reach target value of the conventional "diffusive dose $\mathrm{Kt} / \mathrm{V}$ constituting thus truly combined approach to HDF adequacy in both SM and MM range.

\section{Prescription and efficacy assessment of the convective elimination in HDF}

Hemodiafiltration (HDF) was developed as a more advanced hemoelimination method to fully utilize potential of the highly permeable (high-flux) membranes introduced in the seventies and eighties to remove MM uremic toxins by convection, additionally to diffusion-based elimination of SM catabolites by conventional hemodialysis (HD). The well-known HDF principle is shown in Fig. 1.

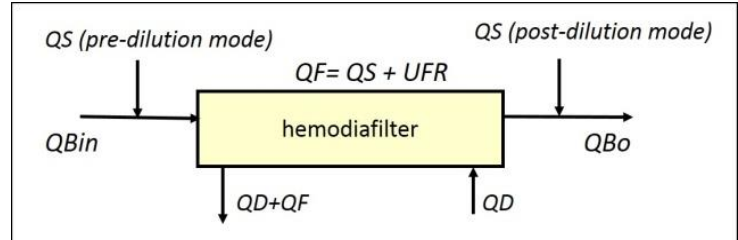

Fig. 1: Principal scheme of pre- and post-dilution mode HDF.

Convective removal rate $(m)$ is given by the product of filtration rate $(Q F)$ and concentration of the given solute in plasma water $(C P)$ in the blood path of the hemodiafilter. The sieving coefficient $S C$ $(S C=C F / C P)$ reflects possible differences between $C P$ and concentration in filtrate $(C F)$.

$$
m=Q F \cdot C P=Q F \cdot C F \cdot S C
$$

Filtration rate $(Q F)$ exceeds significantly patient's ultrafiltration needs $(U F R)$ and that part exceeding $U F R$ is replaced by a continuous inflow of substitution fluid $(Q S)$ in the blood path so that the overall volume balance is preserved:

$$
Q F=Q S+U F R
$$

Addition of substitution fluid at the hemodiafilter blood inflow or outflow defines pre-dilution or post-dilution HDF mode.

As in any other hemoelimination procedure, efficiency of a solute removal in HDF is characterized by the term clearance $(K)$. Physically, $K$ of a certain solute is that fraction of blood flow from which that solute is completely removed during blood passage through the cleansing device, regardless of the physical principle by which the transport of the solute in the device is accomplished. Assuming for simplicity that an MM solute is removed in HDF only by convection (diffusion is neglected) and that its $S C=1, K$ in the postdilutional HDF will be equal to filtration rate $Q F$

$$
K=Q F
$$

and the cleansed volume $\left(V_{\text {conv }}\right)$ will constitute absolute convective dose of the HDF procedure.

$$
V_{\text {conv }}=Q F \cdot t
$$

As a matter of fact, convection occurs in HDF simultaneously with diffusion and both phenomena thus interfere with each other. Due to diffusion, concentration in blood decreases along the hemodiafilter (mainly in SM region) and reduces thus the convective removal, i.e. the amount of solute transported across the membrane with filtrate. Convection realized by the filtrate flow $Q F$ reduces on the other side blood flow and thus diminishes diffusional transport determined mainly by flows. Impact of this interaction on elimination of solutes of different molecular weight is well demonstrated in Fig. 2 adapted from [6]:

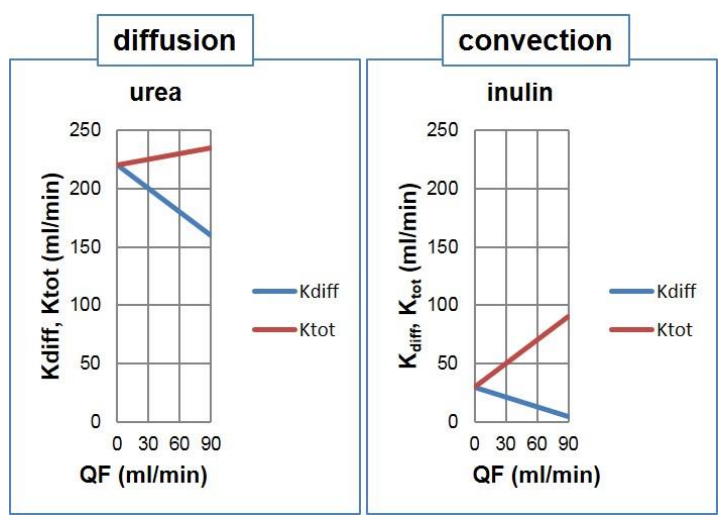

Fig. 2: Interaction between diffusion and convection in postdilutional HDF (adapted from [6]).

In case of urea (mol. weight 60), diffusion decreases significantly with increasing $Q F$ and overall contribution of convection to total clearance $K_{t o t}$ is just marginal. For inulin (mol. weight around 5000), on the contrary, reduction of diffusion with increasing $Q F$ is just small but increase of $K_{t o t}$ is highly significant. Removal quantification of solutes with even higher molecular weight (e.g. $\beta 2$ microglobulin, myoglobin etc.) by means of "convective dose" $V_{\text {conv }}$ is thus well justified.

With definition of the "convective dose" inevitably comes the question of how high it should be. Based on several observational studies which reported decreasing mortality with increasing $V_{\text {conv }}$, part of the nephrological community adopted the approach "the higher the better". Fresenius company as the leader in introducing on-line HDF in clinical practice gave the "hall-mark" to this approach by their concept of "high- 
volume HDF". It was based on three randomized controlled trials - the Dutch CONTRAST study [7], the Turkish study [8], and the Catalonian ESHOL study [5]. All three tried to find out whether the on-line HDF is better than conventional HD primarily in allcause mortality and if so at what $V_{\text {conv }}$ this would become apparent. The CONTRAST study compared HDF with low-flux HD and better all-cause mortality was found only in a post hoc analysis after separation the HDF group in terciles in the highest tercile $\left(V_{\text {conv }}>21.95 \mathrm{~L}\right)$. In the Turkish study (high-flux HD vs. HDF), again in a post hoc analysis only, much lower volume was found $\left(V_{\text {conv }}>17.4 \mathrm{~L}\right)$. In the Catalonian study, post-hoc analysis showed marked improvement in all-cause mortality with the highest volumes $\left(V_{\text {conv }}>25.4 \mathrm{~L}\right)$. Those rather inconsistent results allowed only two general conclusions: The on-line HDF is definitely not inferior to conventional HD and all-cause mortality in HDF patients generally decreases with increasing $V_{\text {conv }}$. None of the three studies provided an upper target value of $V_{\text {conv }}$ above which no further improvement in mortality could be expected and none tried to systematically look for an anthropometric parameter which could be used to define a target relative convective dose of HDF. Nevertheless, the Catalonian study provided at least some data for such a search (supplemental Tab. 4 in [5]). Fig. 3 shows three plots we have created from that Tab. 4 :

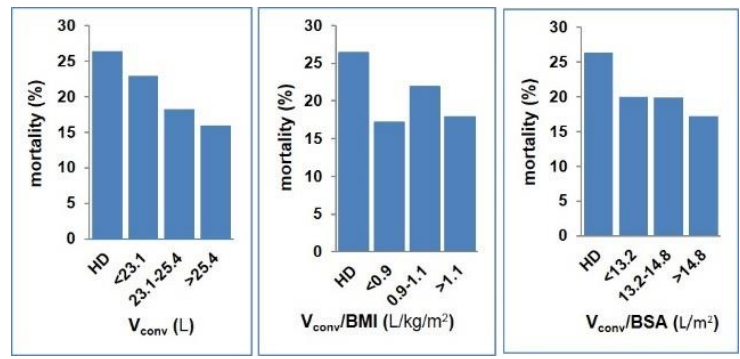

Fig. 3: All-cause mortality in HDF vs. $V_{\text {conv }}, V_{\text {conv }} / B M I$ and $V_{\text {conv }} / B S A$, for comparison also mortality seen in $H D$, adapted from [5].

The left plot shows ever decreasing mortality with increasing absolute $V_{\text {conv }}$. The absolute value thus cannot define the upper limit of $V_{\text {conv }}$ over which no further benefit could be expected (which would mimic adoption of target $s p K t / V=1.4$ after evaluation of the HEMO study [2]). Middle plot displays mortality vs. $V_{\text {conv }}$ normalized to body mass index $(B M I)$. With increasing $V_{\text {conv }} / B M I$, mortality fluctuates, i.e. $B M I$ does not appear a good normalizing parameter either. It is only the right plot of mortality vs. $V_{\text {conv }} / B S A$ which shows flat running pattern-ceasing of mortality decrease for $V_{\text {conv }} / B S A$ over $14-15 \mathrm{~L} / \mathrm{m}^{2}$, suggesting that body surface area $(B S A)$ could well be the sought normalizing parameter. It seems perfectly logical because it is known that metabolic turnover responsible for production of catabolites (to be removed by hemoelimination techniques in renal failure) is proportional to $B S A$ [9]. Also glomerular filtration rate (GFR) used to assess excretorial kidney function has traditionally been normalized to $B S A$, not to total body water $V$. Those were the reasons which lead us to use $V_{\text {conv }} / B S A$ as a marker defining "relative convective dose" in HDF.

Based on the data from the ESHOL study, the target value of $V_{\text {conv }} / B S A$ over which it would have no sense to go because it does not improve mortality any further is estimated as

$$
V_{\text {conv }} / B S A=15 \text { or } V_{\text {conv }}=15 \cdot B S A .
$$

The most often used formula for BSA estimation is that published by Dubois [10]

$$
B S A=0.20247 \cdot H^{0.725} \cdot B W^{0.425}
$$

where $H$ means patient's height in meters and $B W$ his/her body weight (post dialysis) in $\mathrm{kg}$.

Combining Eq. (5) and (6) it is possible to express also the target $V_{\text {conv }}$, needed to fulfil Eq. (5), in terms of $H$ and $B W$, and convert it into a graphic form-see Fig. 4. The plot can be used for an easy prescription of the target $V_{\text {conv }}$.

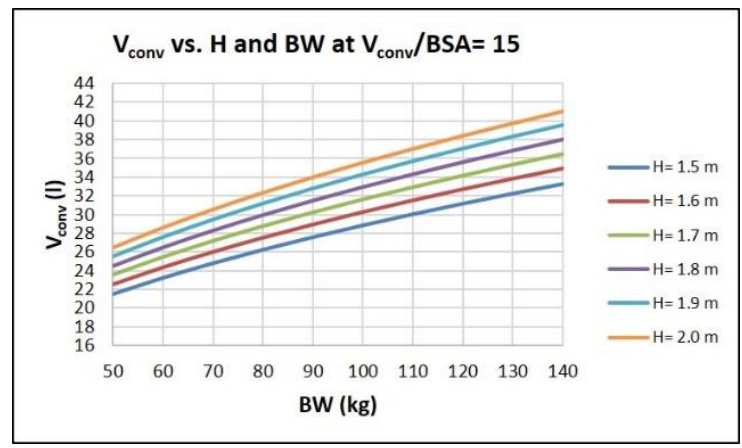

Fig. 4: Plot for $V_{\text {conv }}$ prescription from $H$ and $B W$ fulfilling the requirement $V_{\text {conv }} / B S A=15$.

After defining the target $V_{\text {conv }}$, a question arises how to reach it. Once the $V_{\text {conv }}$ target value is either calculated from Eq. (5) or read from the plot in Fig. 4, it determines filtration rate $Q F=V_{\text {conv }} / t$ where $t$ is the session time (from organisational reasons, the same $t$ value is quite often used in the renal unit for all patients). And $Q F$, in turn, defines the blood flow $(Q B)$ needed to reach that $Q F$ safely, i.e. without excessive risk of blood clotting in the dialyzer because of too high hemoconcentration. The EUDIAL working group (established within the EDTA/ERA to enhance quality of hemoelimination therapies in general) addressed the issue of optimal running parameters of online HDF and suggested in their report [11] that because of the clotting risk, the filtration fraction $(F F)(F F=Q F / Q B)$ should be limited to $20-25 \%$. This is in correspondence with measurement of the fibre bundle volume $(F B V)$ changes during post-dilution HDF [12] done in our unit some time ago-see Fig. 5. 
$F B V$ is the volume of the dialyzer blood path and procedure for its evaluation by means of ultrasonic dilution was described in [13]. The $\triangle F B V(\%)$ denotes its relative change during an HDF session. The plot shows the mean $\triangle F B V$ values obtained from a group of 8 patients hemodiafiltered at identical HDF setting except for the $Q F$ value. As can be seen from the plot, while the change in $F B V$ was negligible with $F F=0.2$ (only $1.07 \%$ ), it increased to over $10 \%$ with $F F=0.3$, indicating loss of functional fibres by deposits and clotting. As far as I know, no such measurement has been made with advanced algorithms for automatic control of $Q F$ aimed at maximization of $V_{\text {conv }}$ (Autosub $^{\text {Plus }}$ of Fresenius or Ultra ${ }^{\text {Control }}$ of Gambro) which usually work at higher $F F$ - up to 0.35 , rather surprisingly without any apparent problems with increased clotting.

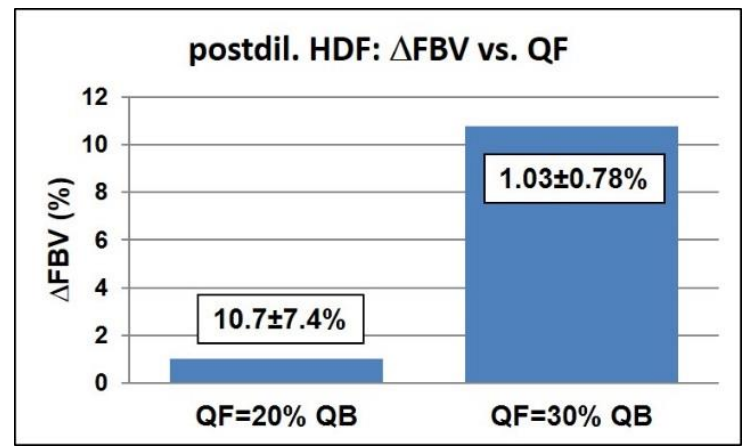

Fig. 5: Reduction in fibre bundle volume during online $H D F$ at $F F=20 \%$ and $30 \%$ (data from the Prague Strahov dialysis unit, FBV measured with the HDO1 device, Transonic Systems).

Considering all the above, value of $F F=0.3$ appears as a reasonable compromise, especially when machines without automatic $V_{\text {conv }}$ control are used. For this $F F$, the formula for $Q B$ allowing to reach prescribed $V_{c o n v}$ (as defined by Eq. (5) or the plot in Fig. 4) can be written:

$$
Q B=Q F / F F=3.3 \cdot Q F=3.3 \cdot V_{\text {conv }} / t
$$

Combining Eq. (7) with Eq. (4), (5) and (6), it is possible to express $Q B$ in terms of patient's $H$ and $B W$ graphically:

$$
\begin{aligned}
Q B= & 3.3 \cdot 1000 \cdot V_{\text {conv }}(L) / t(\min ) \\
= & (3300 \cdot 15 / 240) \cdot 0.20247 \cdot H^{0.725} \\
& \cdot B W^{0.425}
\end{aligned}
$$

( $H$ in $\mathrm{m} ; B W$ in $\mathrm{kg}$, and $t=240 \mathrm{~min}$ ).

In principle, the graph in Fig. 6 is merely a recalculation of Fig. 4. It is, nevertheless, helpful to have both, because in HDF machines without automatic $Q F$ control, it is necessary to set desired value of $V_{\text {conv }}$ and $Q B$ separately. In machines with automatic $Q F$ control, only $Q B$ has to be set, maximized $V_{c o n v}$ is obtained automatically, although its value reached at the end of the session is not known in advance.

Note: Prescription of the $Q B$ value and the $V_{\text {conv }}$ to fulfil the requirement $V_{\text {conv }} / B S A=15$ is valid for postdilution HDF only. For pre-dilution $H D F$, both $Q F$ in Eq. (5) and $V_{\text {conv }}$ read from Fig. 4 must be increased proportionally to the dilution factor $D F=(Q B+Q S) / Q B$.

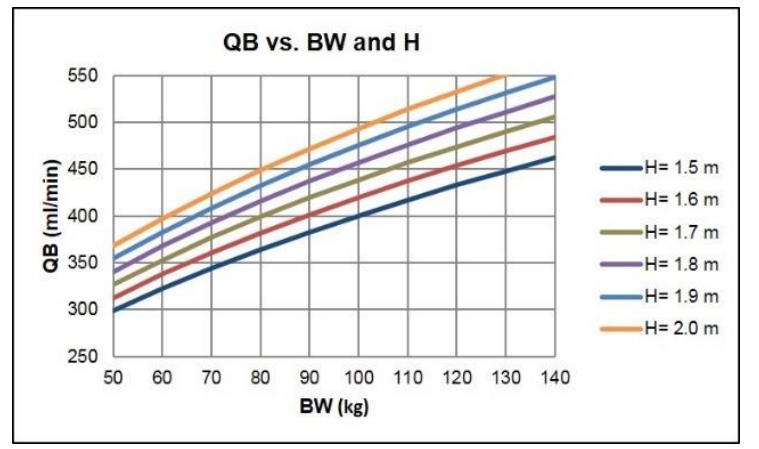

Fig. 6: Plot for prescription of $Q B$ needed to reach $V_{\text {conv }} / B S A=15$ at $F F=0.3$ (calculated for $H D F$ session time $t=4$ hours).

The $Q B$ value defined by the Eq. (8) or Fig. 6 is based on requirements posed on the efficacy of convective elimination of MM catabolites (sufficient $V_{\text {conv }} / B S A$ ). However, $Q B$ is also responsible for sufficient diffusive elimination in the $\mathrm{SM}$ region (adequate $K t / V$ ). If we define the "diffusion-needed" $Q B$ in terms of the same anthropometric parameters as the $Q B$ resulting from consideration of convective transport efficacy, we can see whether it is possible to find a $Q B$ able to ensure sufficient efficacy both in diffusive SM elimination as well as in convective elimination of MM.

\section{Necessary $Q B$ for adequate diffusive elimination in $S M$ region}

Theoretical background of requirement on $Q B$ for HD dose prescription and monitoring $(K t / V)$ has been developed earlier [14]. It is based on two simple assumptions: dialyzer urea clearance $(K)$ is equal to $70 \%$ of blood flow through the dialyzer $(Q B)$ and total body water $(V)$ is equal to $60 \%$ of body weight (post-HD weight). Combining those two assumptions $(K=0.7 \cdot Q B$ and $V=0.6 \cdot B W$ ) with the target $K t / V$ value $K t / V=1.2$ leads to surprisingly simple pre-scription of $Q B$ and monitoring of HD adequacy:

$$
K t / V=0.7 \cdot Q B \cdot t / 0.6 \cdot B W=1.2
$$


Rearranging Eq. (9) gives the formula for prescribing individualized $Q B$ based on patient's $B W$ :

$$
\begin{aligned}
Q B & =K t / V \cdot 0.6 \cdot B W /(0.7 \cdot t) \\
& =1.03 \cdot B W / t
\end{aligned}
$$

Eq. (10) can be displayed in a graphic form of $Q B$ vs. $B W$ with session time $t$ as a parameter. Resulting plot is shown on Fig. 7.

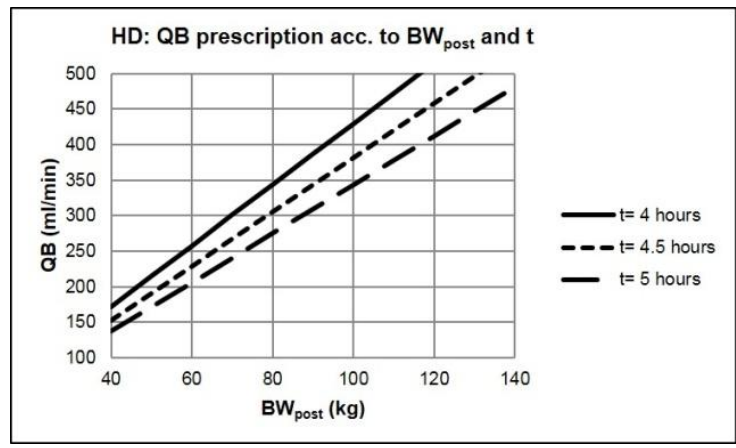

Fig. 7: Prescribed $Q B$ vs. $B W$ and $t(K t / V=1.2$; $K=0.7 Q B ; V=0.6 B W$ ) according to $E q .(10)$.

The plot was calculated for $K t / V=1.2$ but can be easily modified using Eq. (9) and (10) if a $K t / V$ different from 1.2 should be obtained.

Rearrangement of Eq. (9) then provides a simple and elegant approach to estimation of the delivered $K t / V$ at the end of HD: In Eq. (9), $Q B \cdot t$ actually gives the value of total blood volume processed during HD $(V B)$, a parameter displayed today by practically all dialysis machines. A formula to estimate delivered $K t / V$ in terms of $V B / B W$ can thus be devised:

$$
\begin{aligned}
K t / V & =0.7 \cdot V B /(0.6 \cdot B W) \\
& =1.17 \cdot V B / B W
\end{aligned}
$$

Eq. (11) actually says that $K t / V$ of 1.2 is delivered if the processed blood volume roughly equals body weight. Very simple rule without any need for blood sampling! Practical verification of $Q B$ prescription according to $B W$ and delivered $K t / V$ estimation from the $V B / B W$ value has been done in a larger patient cohort and resulted in sensitivity and specificity of inadequate $K t / V$ detection $(K t / V<1.2) 75 \%$ and $85 \%$, respectively $[15,16]$-see Fig. 8.

Minor discrepancies from good correspondence between conventionally measured $K t / V$ and $V B / B W$ (the right bottom quadrant and the left upper quadrant) were seen in patients with higher $B M I$ (the left upper quadrant of the plot, i.e. with $V<0.6 \cdot B W)$ or in malnourished patients with low $B M I$ (the right bottom quadrant, i.e. $V>0.6 \cdot B W)$.

Note: The target value $K t / V=1.2$ in the above calculations was used because the in vivo data was obtained on dialysis machines with the online $\mathrm{Kt} / \mathrm{V}$ evaluation which gives $K t / V$ values closer to equilibrated $K t / V(e K t / V)$ rather than to single-pool $K t / V(\operatorname{spK} / V)[3,4]$.

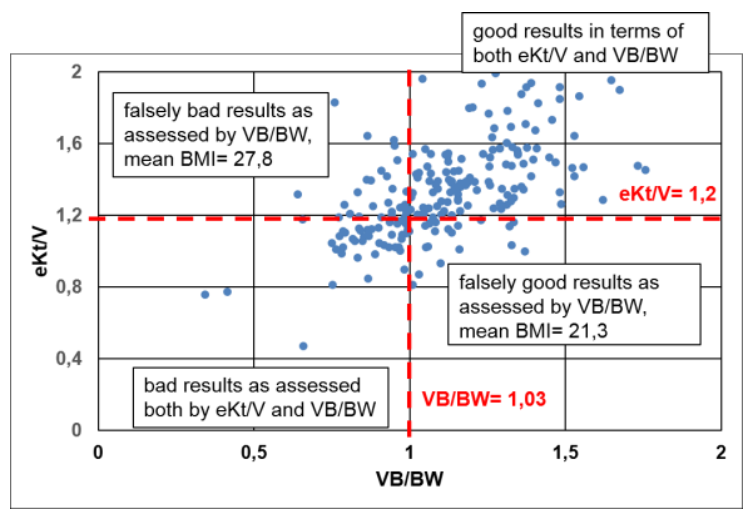

Fig. 8: In vivo eKt/V vs. $V B / B W$ relation-influence of anthropometric factors [16] (data from the Prague Strahov dialysis unit).

\section{Combined prescription and efficacy assessment in HDF}

As has been shown in the above two paragraphs, the prescribed $Q B$ affects treatment efficacy both in the SM (diffusion-based transport) and MM region (convective transport), although in both cases via different mechanisms. $Q B$ thus has a "double function". With defined $Q B$ value needed to achieve adequate dialysis in SM region characterized by the $K t / V$ value and $Q B$ needed to reach sufficient $V_{\text {conv }} / B S A$ as the adequacy marker in MM region, it is possible to look whether a common $Q B$ can be found which would fulfil both requirements, i.e. $K t / V=1.2$ and $V_{\text {conv }} / B S A=15$ in a particular patient.

According to Eq. (10), the $Q B$ value needed to reach the target $K t / V$ value at a given $t$ depends primarily on patient's body weight. Assuming a technically feasible filtration fraction $F F=Q F / Q B=0.3$, it is possible to calculate obtainable value of $V_{\text {conv }}$ at a given $Q B$ combining Eq. (7) and Eq. (11):

$$
\begin{aligned}
V_{\text {conv }} & =Q F \cdot t=0.3 \cdot Q B \cdot t \\
& =0.3 \cdot 1.03 \cdot B W=0.309 \cdot B W
\end{aligned}
$$

This formula represents a straight line in a plot of $V_{\text {conv }}$ vs. $B W$ such as the plot in Fig. 4. Adding the line corresponding to Eq. (12) in the plot at Fig. 4 (read dashed line), a modified plot is obtained (Fig. 9), enabling combined approach to $V_{\text {conv }}$ prescription to fulfil the target values of both diffusion-based adequacy marker $K t / V$ and the novel convection-based adequacy marker $V_{\text {conv }} / B S A$.

Due to the higher slope of the red dashed line obtained from Eq. (12) for increasing $B W$, it intersects the 
lines $V_{\text {conv }}$ vs. $B W$ calculated by Eq. (8). In the red area left from the intersection region ( $B W$ below cca $85 \mathrm{~kg}$ ), $Q B$ prescription needed to reach target $K t / V$ would not enable to reach $V_{\text {conv }} / B S A=15$ and $Q B$ must be prescribed according to Eq. (8) giving priority to the "convective dialysis dose". In the middle green region, prescription of the $Q B$ both according to Eq. (12) and Eq. (8) would roughly enable to reach both the target $K t / V$ and the $V_{\text {conv }} / B S A$. On the contrary, in the largest body size patients (for $B W$ over cca $125 \mathrm{~kg}$ ), the target "convective dose" would be reached but the "diffusive dose" would not, i.e. in that region, $Q B$ should be prescribed according to Eq. (12). This discordance in $Q B$ prescription would partly be solved by the novel requirement for a non-uniform $K t / V$ value which would account for gender and body size-see the Discussion paragraph below.

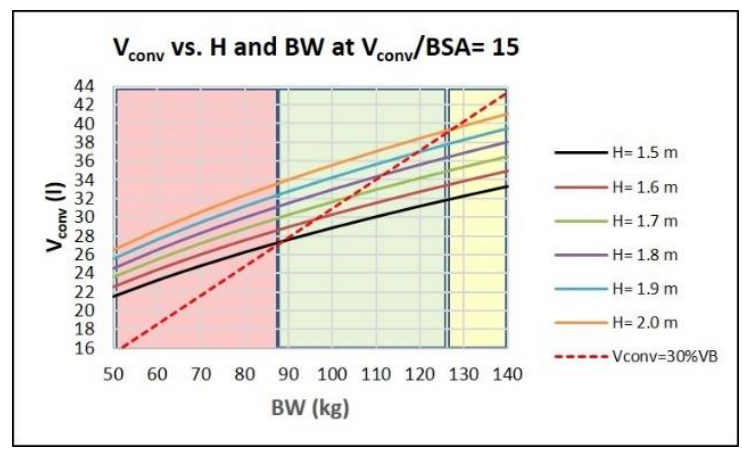

Fig. 9: Combined approach to prescription of adequate $H D F$.

\section{Discussion}

The above described novel combined procedure is at present merely a hypothesis how the issue of HDF prescription and adequacy assessment could be practically done. Suitability of the $V_{\text {conv }} / B S A$ as a convective adequacy marker, whatever intuitively logical, is at present supported only by limited amount of clinical data taken from the ESHOL study. Thus, larger statistically sufficiently powered studies will be needed to confirm the suggested approach.

The problem of discordance between the $Q B$ needed to reach both adequate $K t / V(=1.4$ or 1.2 , respectively) and adequate $V_{\text {conv }} / B S A(=15)$ is a consequence of different normalizing parameter in both cases $-V$ in the former and $B S A$ in the latter. As a matter of fact, after about two decades of enthusiastic use of $K t / V$ when a lot of observational studies documented close association of $K t / V$ with all-cause mortality, some discrepancies appeared which pointed towards problems with its universal target value for all patients. Owen [17] detected in his study gender dependence of an adequate relative dialysis dose (for the same survival, the $K t / V$ needed in females was higher than that in males). Wolfe [18] found the same for small and large body size patients - smaller patients needed higher $K t / V$ than the larger ones. While the two above mentioned studies were just observational, their conclusions were subsequently confirmed by the large randomized controlled HEMO study [19, 20]-female and small patients indeed needed higher $K t / V$ than males and patients with a larger body size. Several researchers tried to solve the problem of adjusting the $K t / V$ to patient's gender and/or body characteristics by using a different parameter for $K t$ normalisation. The so far most elaborated approach has been offered by Daugirdas [21] using the ratio of total body water $V$ and body surface area $B S A$ to define a modified $K t / V(\bmod K t / V)$ :

$$
\begin{aligned}
\operatorname{modKt} / V= & (\text { conventional Kt } / V) \\
& \cdot(V / B S A)_{\text {ref }} /(V / B S A)_{\text {pat }}
\end{aligned}
$$

He analyzed the huge patient data pool from the HEMO study and suggested a gender and body sizedependent equation of the ratio $V / B S A$. As the reference value of the $V / B S A$ ratio $(V / B S A)_{r e f}$, he used the mean value from the whole patient cohort in the HEMO study (over 1700 patients) $-20 \mathrm{~L} / \mathrm{m}^{2}$.

Fig. 10 shows the mod $K t / V$ vs. $B W$ using Eq. (13) separately for males and females of different $B W$ and $H$, calculating $B S A$ according to Dubois Eq. (6) and total body water $V$ (in liters) according to Watson's formulas [22] assuming conventional $s p K t / V=1.4$ (dashed red line, Fig. 10).

For males:

$$
\begin{aligned}
V= & 2.447-0.09156 \cdot A+0.1074 \cdot H \\
& +0.3362 \cdot B W
\end{aligned}
$$

For females:

$$
V=0.1069 \cdot H+0.2446 \cdot B W-2.097
$$

with $A$ being patient's age (years), $H$ his/her height (cm) and $B W$ his/her body weight $(\mathrm{kg})$.

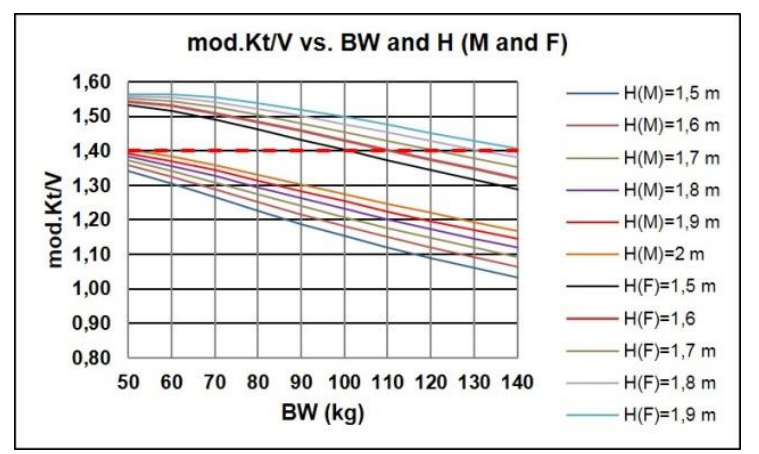

Fig. 10: ModKt/V calculated by Eq. (13) vs. BW calculated for conventional spKt/V $=1.4$ and $V / B S A)_{\text {ref }}=15$.

The dashed red line in the plot represents the current uniform $s p K t / V$ value 1.4. The upper set of curves represents females with height $H(F)$ from 1.5 to $1.9 \mathrm{~m}$. 
Only females with large body size need lower $K t / V$ than the currently used target $(s p K t / V=1.4, e K t / V=1.2)$, smaller females, on the contrary, need higher $K t / V$. As for males (the bottom set of curves with height $H(M)$ from 1.5 to $2.0 \mathrm{~m}$ ), target $\mathrm{modKt} / \mathrm{V}$ has the same value as is the current target, while the largest body size males would need significantly less. The $\operatorname{modKt} / V$ thus accounts for both previously published objections against a uniform $K t / V$ for all patients. And in fact, this modified approach to "diffusive dose" $K t / V$ would also partly solve the discordance between the $Q B$ needed to reach sufficient diffusional modKt/V and $Q B$ calculated from the desirable $V_{\text {conv }}$. The slope of the $K t / V$-derived line (red dashed line in Fig. 9) would become less steep and would thus get closer to curves generated from the condition $V_{\text {conv }} / B S A=15$. It means that application of the modified $K t / V$ approach would lead to better correspondence in $Q B$ needed for sufficient diffusional and for convective elimination efficacy of HDF.

\section{Summary and conclusions}

In online HDF, treatment dose in SM (diffusionbased dose) and MM range (convective dose) are defined separately. Surrogate of the relative diffusionbased dose has traditionally been $K t / V$ with a uniform target value. Absolute convective dose of postdilutional HDF is well defined by the convective volume $V_{\text {conv }}$, although there is no generally accepted target value of it. There is also no consensus on an anthropometric parameter for conversion of absolute "convective dose" to a relative one and hence also no accepted target value of it either. Based on generally assumed direct proportionality between metabolic turnover and limited amount of data from the ESHOL study, this article suggests introduce $V_{\text {conv }} / B S A$ as the relative convective dose with a target value of $15 \mathrm{~L} / \mathrm{m}^{2}$.

For practical application of the above suggestion, use of the plots in Fig. 4 and Fig. 6 is sufficient. First, the $V_{\text {conv }}$ value read from the plot at Fig. 4 for the $B S A$ ( $H$ and $B W$ ) of a given patient is prescribed and subsequently necessary $Q B$ value to reach that $V_{\text {conv }}$ is read from Fig. 6 and set on the HDF machine. In machines with automatic control of $Q F$ via the filtration fraction $\mathrm{FF}$, only the $Q B$ needs to be defined prior to HDF start and the FF value set to 0.3. Even in HDF machines equipped with automated maximisation of $V_{c o n v}$, which do not need $V_{\text {conv }}$ prescription, the suggested concept of $V_{\text {conv }} / B S A$ can still be used to assess adequacy of $V_{\text {conv }}$ reached.

There is certain dissonance between the $Q B$ needed to reach adequate $K t / V(\operatorname{spKt} / V=1,4$ or $e K t / V=1,2$ in thrice weekly schedule) and $Q B$ needed to reach adequate value of the newly suggested marker of "convective dose" $V_{\text {conv }} / B S A$ - see Fig. 9. The $Q B$ value derived from the target value of the conventional $K t / V$ is lower than the $V_{c o n v}$-derived $Q B$ in patients with $B W$ up to cca $85 \mathrm{~kg}$. Hence the $Q B$ prescribed for an adequate HDF in smaller patients should be $V_{\text {conv }}$-derived. In the largest body size patients ( $B W$ over $125 \mathrm{~kg})$, the opposite is true.

The above-mentioned dissonance in $Q B$ prescription based on "diffusive dose" $(K t / V)$ and on "convective dose" $\left(V_{\text {conv }} / B S A\right)$ in HDF would be less pronounced with the $\operatorname{modKt} / V$ concept than with the conventional uniform $K t / V$ approach. The newly suggested concept of the relative convective dose $V_{\text {conv }} / B S A$ is at present not supported by sufficient clinical data and larger randomized controlled studies investigating its association with mortality and other "softer" treatment outcomes (hospitalisation rate, ESA needs etc.) are thus warranted. To facilitate use of the $V_{\text {conv }} / B S A$ and associated $Q B$ approach, an Excel file is attached to the electronic version of this article to perform all necessary calculations.

The absolute convective volume per se can, of course, be very well used for studies on elimination of any specific MM uremic toxin once its sieving coefficient is known.

\section{Declaration of conflicting interests}

There is no conflict of interest to declare.

\section{References}

[1] Gotch FA, Sargent JA. A mechanistic analysis of the National Cooperative Dialysis Study (NCDS). Kidney Int. 1985;28:52634. DOI: $\underline{10.1038 / \mathrm{ki} .1985 .160}$

[2] Eknoyan G, Beck GJ, Cheung AK, Daugirdas JT, Greene T, Kusek JW, et al. Effect of dialysis dose and membrane flux in maintenance hemodialysis. New Engl J Med. 2002;347(25): 2010-19. DOI: 10.1056/NEJMoa021583

[3] KDOQI Clinical practice guideline for hemodialysis adequacy: 2015 update. Am J Kidney Dis. 2015;66(5):884-930. DOI: $10.1053 /$ j.ajkd.2015.07.015

[4] Eureopean Best Practice Guidelines: II.1 Haemodialysis dose quantification: small solutes. Nephrol Dial Transplantat. 2002; 17(Suppl 7):17-21.

[5] Maduell F, Moreso F, Pons M, Ramos R, Mora-Macià J, Carreras J, et al, ESHOL Study Group. High-efficiency postdilution online hemodiafiltration reduces all-cause mortality in hemodialysis patients. J Am Soc Nephrol. 2013;24(3): 487-97. DOI: 10.1681/ASN.2012080875

[6] Ledebo I, Blankenstijn P. Haemodiafiltration-optimal efficiency and safety. Nephrol Dial Transplant Plus. 2010;3(1):816. DOI: $10.1093 /$ ndtplus/sfp149

[7] Grooteman MP, van den Dorpel MA, Bots ML, Penne EL, van der Weerd NC, Mazairac AH, et al, CONTRAST Investigators. Effect of online hemodiafiltration on all-cause mortality and cardiovascular outcomes. J Am Soc Nephrol. 2012;23:108796. DOI: $10.1681 / A S N .2011121140$

[8] Ok E, Asci G, Toz H, Ok ES, Kircelli F, Yilmaz M, et al. Turkish Online Haemodia-filtration Study: Mortality and cardiovascular events in online haemodiafiltration (OL-HDF) 
compared with highflux dialysis: results from the Turkish OLHDF Study. Nephrol Dial Transplant. 2013;28:192-202.

[9] White CR, Seymour RS. Allometric scaling of mammalian metabolism. J Exp Biol. 2005;208:1611-9. DOI: $10.1242 / \mathrm{jeb} .01501$

[10] Dubois D, Dubois EF. A formula to estimate the approximate surface area if height and weight be known. Arch Intern Med. 1916;17:863-71.

[11] Tattersall JE, Ward RA (Eudial group). Online haemodiafiltration: definition, dose quantification and safety revisited. Nephrol Dial Transplant. 2013;28:542-50. DOI: 10.1093/ndt/gfs530

[12] Slavičková E. Dialyzer fibre bundle volume measurement during dialysis, MSc dipl. thesis, Healthcare studies, $1^{\text {st }}$ Faculty of Medicine, Charles University, Prague, 2006 (in Czech).

[13] Krivitski NM, Kislukhin VV, Snyder JW, MacGibbon DR, Kuznetsova OA, Reasons AM, Depner TA. In vivo measurement of hemodialyzer fiber bundle volume: Theory and validation. Kidney Int. 1998;54:1751-8. DOI: $10.1046 / \mathrm{j} .1523-1755.1998 .00146 . x$

[14] Kesziová A, Kinská H, Švárová B, Nejedlý B, Lopot F. Are the blood flow and cumulative blood volume suitable control and monitoring parameters of dialysis dose? EDTNA/ERCA J. 2003;29(3):118-22.

[15] Lopot F, Bláha J, Nejedlý B, Polakovič V. Analysis of cumulative blood volume-based $K t / V$ prescription and monitoring of its delivery. Int J Artif Organs. 2006;29(5):501 (abstract).

[16] Lopot F, Bláha J, Švára F, Polakovič V. A simple method for prescription and monitoring of dialysis dose $K t / V$. Aktuality v nefrologii. 2007;2:57-61 (in Czech, abstract in English).

[17] Owen WF Jr, Chertow GM, Lazarus JM, Lowrie EG. Dose of hemodialysis and survival: differences by race and sex. J Am Med Assoc. 1998;280:1764-8. DOI: $10.1001 /$ jama.280.20.1764
[18] Wolfe RA, Ashby VB, Daugirdas JT, Agodoa LY, Jones CA, Port FK. Body Size, Dose of Hemodialysis, and Mortality. Am J Kidney Dis. 2000;35(1):80-8.

DOI: $10.1016 / \mathrm{S} 0272-6386(00) 70305-2$

[19] Depner T, Daugirdas J, Greene T, Allon M, Beck G, Chumlea $\mathrm{C}$, et al. Dialysis dose and the effect of gender and body size on outcome in the HEMO study. Kidney Int. 2004;65(4):1386-94. DOI: 10.1111/j.1523-1755.2004.00519.x

[20] Greene T, Daugirdas J, Depner T, Allon M. Beck G, Chumlea $\mathrm{C}$, et al. Association of achieved dialysis dose with mortality in the Hemodialysis study: An example of "dose-targeting bias". J Am Soc Nephrol. 2005;16(11):3371-80. DOI: 10.1681/ASN.2005030321

[21] Daugirdas JT, et al. $K t / V$ rescaling to $B S A$ - implications for different-size patients by gender. Sem Dial. 2008,21:415-21.

[22] Watson PE, Watson ID, Batt RD. Total body water volumes for adult males and females estimated from simple anthropo-metric measurements. Am J Clin Nutr. 1980;33(1):27-39. DOI: $\underline{10.1093 / a j e n / 33.1 .27}$

František Lopot, PhD.

General University Hospital, Dpt. Medicine Šermírská 4, 16900 Prague 6, Czech Republic

E-mail: f.lopot@email.cz Phone: +420225003206 